\title{
Correction to: Epidemiology and Racial/Ethnic Differences in Food Allergy
}

Jialing Jiang, Christopher M. Warren, and Ruchi S. Gupta

\section{Correction to: Chapter 1 in: R. S. Gupta (ed.), Pediatric Food Allergy, https://doi.org/10.1007/978-3-030-33292-1_1}

A typographical error in the chapter has been corrected. The line "Specifically, peanut allergy was more prevalent among Jewish infants..." has now been changed as "Specifically, peanut allergy was less prevalent among Jewish infants..." 